\title{
Measuring joint kinematics of treadmill walking and running: comparison between an inertial sensor based system and a camera-based system
}

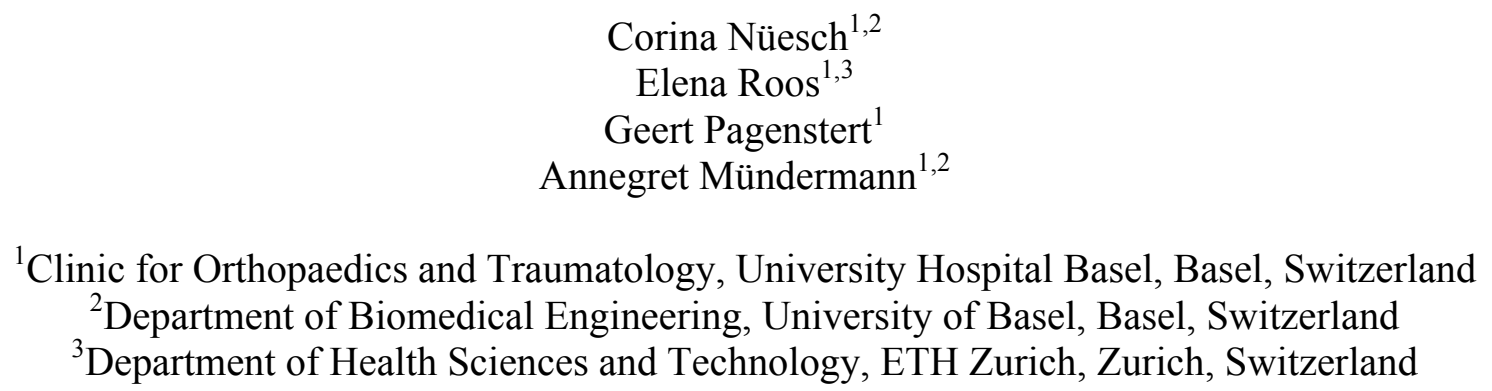

${ }^{1}$ Clinic for Orthopaedics and Traumatology, University Hospital Basel, Basel, Switzerland ${ }^{2}$ Department of Biomedical Engineering, University of Basel, Basel, Switzerland ${ }^{3}$ Department of Health Sciences and Technology, ETH Zurich, Zurich, Switzerland

Original Article

Nüesch C., E. Roos, G. Pagenstert, and A. Mündermann (2017) Measuring joint kinematics of treadmill walking and running: comparison between an inertial sensor based system and a camera-based system. Journal of Biomechanics 57:32-38. DOI:

10.1016/j.jbiomech.2017.03.015. This work is licensed under a Creative Commons Attribution 4.0 International License.

Address for Correspondence:

Dr. Corina Nüesch

Clinic for Orthopaedics and Traumatology

University Hospital Basel

Spitalstrasse 21

4031 Basel, Switzerland

Tel. +41612659444

Email corina.nueesch@usb.ch

Key words: inertial sensors; kinematics; walking; running; agreement

Word count: 3419 (Abstract: 250) 


\section{Abstract}

Inertial sensor systems are becoming increasingly popular for gait analysis because their use is simple and time efficient. This study aimed to compare joint kinematics measured by the inertial sensor system RehaGait ${ }^{\circledR}$ with those of an optoelectronic system $\left(\right.$ Vicon $^{\circledR}$ ) for treadmill walking and running. Additionally, the test re-test repeatability of kinematic waveforms and discrete parameters for the RehaGait ${ }^{\circledR}$ was investigated. Twenty healthy runners participated in this study. Inertial sensors and reflective markers (PlugIn Gait) were attached according to respective guidelines. The two systems were started manually at the same time. Twenty consecutive strides for walking and running were recorded and each software calculated sagittal plane ankle, knee and hip kinematics. Measurements were repeated after 20 minutes. Ensemble means were analyzed calculating coefficients of multiple correlation for waveforms and root mean square errors (RMSE) for waveforms and discrete parameters. After correcting the offset between waveforms, the two systems/models showed good agreement with coefficients of multiple correlation above 0.950 for walking and running. RMSE of the waveforms were below $5^{\circ}$ for walking and below $8^{\circ}$ for running. RMSE for ranges of motion were between $4^{\circ}$ and $9^{\circ}$ for walking and running. Repeatability analysis of waveforms showed very good to excellent coefficients of multiple correlation $(>0.937)$ and $\mathrm{RMSE}$ of $3^{\circ}$ for walking and $3^{\circ}$ to $7^{\circ}$ for running. These results indicate that in healthy subjects sagittal plane joint kinematics measured with the RehaGait ${ }^{\circledR}$ are comparable to those using a Vicon ${ }^{\circledR}$ system/model and that the measured kinematics have a good repeatability, especially for walking.

60 


\section{Introduction}

Gait analysis is an important tool for objectively assessing gait function by providing information on spatiotemporal parameters (e.g. step length, step time, length of stance phase) and lower extremity joint kinematics, kinetics and muscle activation. However, conventional instrumented three-dimensional gait analyses with simultaneous measurements with cameras, force plates and electromyography is costly and time consuming. Technological advances have facilitated development of alternatives to such laboratory based analyses. In recent years, the popularity of inertial sensor based motion analysis systems for assessing joint kinematics has increased (Hamacher et al., 2014; Sprager and Juric, 2015) with the advantage of simple and time efficient gait analyses outside of the laboratory environment.

For instance, the RehaGait ${ }^{\circledR}$ system/model includes seven inertial sensors and software that calculates spatiotemporal parameters and sagittal ankle, knee and hip kinematics. This system has good reliability for spatiotemporal variables and the minimal foot-to-ground angle with intraclass correlation coefficients (ICC) between 0.874 and 0.948 (Schwesig et al., 2010). Spatiotemporal variables measured using an inertial sensor system showed good agreement with those measured using an instrumented treadmill with average ICCs above 0.897 (Donath et al., 2016). Similar data on comparison of kinematic data of the RehaGait ${ }^{\circledR}$ system/model and of an optoelectronic system/model during walking and running are currently lacking.

The concurrent validity of kinematic data presumably depends on the specific combination of inertial sensors and models. Initial results for other inertial sensor based systems/models were promising where kinematic data measured from an inertial sensor system and kinematic data measured through marker clusters at the same position as the inertial sensor were interchangeable (e.g. "Outwalk” or "Cast” with Xsens ${ }^{\circledR}$ or Vicon ${ }^{\circledR}$; coefficient of multiple correlation for sagittal ankle, knee and hip kinematics $>0.95$ ) (Ferrari et al., 2010b). The results were even better when the offset between the systems/models was 
corrected. Moreover, high correlations between calculated joint angles of another system compared to the ones of a marker based model were reported $(>0.80)$ for the sagittal knee and hip angle, but correlations were low $(<0.10)$ for the sagittal ankle angle during walking at normal speed (Cloete and Scheffer, 2008). The reported average root mean squared errors (RMSE) in the sagittal plane ranged from $10^{\circ}$ to $20^{\circ}$ for the calculated data and from $5^{\circ}$ to $12^{\circ}$ after correcting the offset (Cloete and Scheffer, 2008). In contrast, another study (Picerno et al., 2008) reported small differences $\left(\mathrm{RMSE}<5^{\circ}\right)$ for three-dimensional ankle, knee and hip kinematics during walking between inertial and magnetic sensors combined with an anatomical landmark calibration and a marker based model.

The primary aim of this study was to compare the joint kinematics measured by the inertial sensor system RehaGait ${ }^{\circledR}$ with those of a commonly used clinical optoelectronic protocol for treadmill walking and running. We hypothesized that the sagittal plane kinematics of the two systems/models would be highly correlated and that there would be no differences between discrete parameters (minimum/maximum values, range of motion) calculated from the kinematic waveforms of the two systems/models. The secondary aim of the study was to investigate the test-retest repeatability of the kinematic waveforms and the discrete parameters measured by the inertial sensor system/model.

\section{Methods}

\section{Participants}

Twenty healthy subjects (12 female; age: $27.4 \pm 8.3$ years; height: $1.75 \pm 0.08 \mathrm{~m}$; body mass: $66.5 \pm 12.5 \mathrm{~kg}$; body mass index: $21.5 \pm 2.5 \mathrm{~kg} / \mathrm{m}^{2}$ ) participated in this study. Exclusion criteria were pain and/or lower leg injuries within the last 6 months. All subjects were experienced runners with a weekly mileage of $45 \pm 20 \mathrm{~km} /$ week. The study was approved by the local ethical committee and all subjects signed informed consent forms prior to participation. 
Procedures and data processing

All subjects performed a walking and running analysis at their self-selected comfortable

117 speed on an instrumented treadmill (hp/cosmos mercury; Zebris, Isny, Germany) wearing

118 their preferred running shoe. Kinematic data were collected using two independent systems

119 and models - inertial sensor based and optoelectronic based - that were manually started at

120 the same time.

Inertial sensor system and model. The inertial sensor system (RehaGait ${ }^{\circledR}$, Hasomed,

Magdeburg, Germany) consists of seven inertial sensors each comprising a triaxial

accelerometer $( \pm 16 \mathrm{~g})$, a triaxial gyroscope $( \pm 2000 \% \mathrm{~s})$ and a triaxial magnetometer $( \pm 1.3$

Gs). The sensors were placed on the sacrum and bilaterally on the lateral thigh (middle), lateral shank (lower third), and lateral foot (on the shoe, below lateral malleolus) using double sided tape and elastic straps (Figure 1). The manufacturer's software and model was used to calculate ankle, knee and hip angles in the sagittal plane with a sampling frequency of 400 Hz. The system and model are calibrated while the subject is in a neutral upright standing position for $10 \mathrm{~s}$ and performs a slight squatting movement according to the manufacturer's instructions. Hip extension is defined as positive and hip flexion as negative angles, and hence all hip angles were multiplied by -1 to be consistent with the calculated angles from the optoelectronic reference system.

Optoelectronic system and model. The optoelectronic system consisted of a 6-camera motion analysis system (Vicon MX, Vicon Motion Systems Ltd., Oxford, UK) and 16 reflective markers that were placed on anatomical landmarks according to the PlugIn Gait

138 model - bilaterally on the posterior superior iliac spine, anterior superior iliac spine, lateral thigh, lateral epicondyle of the knee, lateral shank, lateral malleolus, heel and second 
metatarsal head (Kadaba et al., 1990). The infrared cameras tracked three-dimensional marker

141 positions with a sampling frequency of $200 \mathrm{~Hz}$. The Nexus software and PlugIn Gait model

142 (Version 1.8.5, Vicon Motion Systems Ltd., Oxford, UK) were used to calculate three-

143 dimensional kinematics of the ankle, knee and hip joint. A static calibration trial in neutral

144 upright standing position was recorded before the dynamic walking and running trials.

After all sensors and markers were attached to the lower extremity, subjects first walked on the treadmill for $30 \mathrm{~s}$ at their self-selected comfortable walking speed (for walking 1 hour).

148 Subsequently, data collection was initiated and kinematic data were recorded simultaneously

149 with both systems for 20 consecutive walking strides. The treadmill speed was then increased to the self-selected running speed (comfortable running speed for 45 minutes) and subjects ran for 3 minutes to adopt their regular running style before kinematic data were recorded with both systems for 20 consecutive running strides (right foot strike to right foot strike). inertial sensor placement and measurement procedure was repeated for walking and running after 20 minutes.

\section{Data analysis}

The recorded waveforms for all sagittal plane kinematics of the ankle, knee and hip

159 joint for both measurement system/models were cut into strides by defining the minimum

160 knee angle after the swing phase as initial contact for both walking and running (Fellin et al.,

1612010 ). All strides were time normalized to 0 to $100 \%$ beginning and ending at initial contact.

162 For each subject, system and joint, the ensemble means of angle waveforms and of peak joint

163 angles of 20 strides were calculated and used for further analysis. Discrete parameters were

164 calculated for the 20 strides of the two measurement systems/models as follows (Figure 2): 
ankle angle, difference between the maximal and the first minimal ankle angle (dorsiflexion

167 range of motion), difference between the maximal and the second minimal ankle angle

168 (plantarflexion range of motion), knee joint angle at initial contact, first maximal knee joint

169 angle, second maximal knee joint angle, minimal knee angle between the first and second

170 maximum, difference between the first maximal and the minimal knee angle (range of motion

171 first half stride), difference between the second maximal and the minimal knee angle (range of

172 motion second half stride), hip angle at initial contact, minimal hip angle, first maximal hip

173 angle, second maximal hip angle, difference between first maximal and minimal hip angle

174 (range of motion first half stride), and difference between minimal and second maximal hip

175 angle (range of motion second half stride).

Statistical analysis

All statistical analyses were performed in SPSS version 22.0 (IBM Corporation, Armonk, NY) and Matlab (Version 2010a, MathWorks Inc., Natick, MA). To compare the joint kinematics calculated from the RehaGait ${ }^{\circledR}$ system with the reference system the following parameters were calculated: RMSE and coefficient of multiple correlation (Ferrari et al., 2010a). RMSE of the waveforms was calculated with the ensemble mean data for each subject and then averaged across joint and condition. The following interpretation of coefficient of multiple correlation was used (Ferrari et al., 2010b): weak $(<0.65)$; moderate (0.65-0.75); good (0.75-0.85); very good (0.85-0.95): excellent $(>0.95)$. This analysis was repeated after removing the offset between the kinematic waveforms of the two systems/models by centering each waveform on its respective mean (i.e. subtracting the mean of a waveform from the entire waveform). The same parameters were calculated for the test re-test repeatability of the RehaGait ${ }^{\circledR}$ system/model. Additionally, ICC with a two-way random model for consistency and the systematic bias (mean difference between measurements) with $95 \%$ limits of agreement $(1.96 *$ standard deviation of the difference 
between measurements) depicted as Bland and Altman plots were calculated for the ranges of motion in walking and running. ICC were rated as excellent $(0.9-1)$, good $(0.74-0.89)$, moderate (0.4-0.73), and poor (0-0.39) (Fleiss, 1986).

To reduce the complexity of the statistical analyses, only data of the right limb were analyzed. Statistically significant differences in discrete kinematic parameters between systems and models were detected using general linear models with factors time and system and with Bonferroni correction to account for multiple parameters (significance level alpha: $0.050 / 18=0.003)$ with least square distance post hoc tests.

\section{Results}

\section{Walking}

The mean self-selected walking speed was $1.37 \pm 0.13 \mathrm{~m} / \mathrm{s}$. There was a good agreement between the average kinematic waveforms measured with the RehaGait ${ }^{\circledR}$ and the reference system/model with very good to excellent coefficients of multiple correlation (Figure 2). Removing the offset between the kinematic waveforms of the two systems/models resulted in excellent coefficients of multiple correlation for all joints (between 0.967 and 0.988). The average RMSE between the original waveforms measured by the two systems $/$ models was smaller than $5^{\circ}$ for the ankle joint and between $7^{\circ}$ and $9^{\circ}$ for the knee and hip joint. After offset correction, the RMSE was smaller than $5^{\circ}$ for all joints (Table 1 ).

The RMSE of the discrete parameters between the RehaGait ${ }^{\circledR}$ and the reference system/model ranged from $4^{\circ}$ to $9^{\circ}$ for the ranges of motion and from $4^{\circ}$ to $15^{\circ}$ for the other parameters (Table 2). For the ankle joint the RehaGait ${ }^{\circledR}$ system/model measured significantly greater plantarflexion after initial contact and a significantly greater range of motion in the stance phase than the reference system/model, while the other parameters showed no statistically significant differences. Knee flexion angle at initial contact and peak knee flexion angle during stance were significantly smaller and range of motion during swing significantly 
218 greater with the RehaGait ${ }^{\circledR}$ than with the reference system/model. For the hip joint, all

219 discrete parameters were significantly different between the two systems/models (Figure 3, 220 Table 3).

\section{Running}

The self-selected running speed was on average $2.93 \pm 0.35 \mathrm{~m} / \mathrm{s}$. For running, the coefficient of multiple correlation between the knee kinematics measured with the RehaGait ${ }^{\circledR}$ system/model and the reference system/model was very good, while the coefficient of multiple correlation was moderate for the ankle kinematics and weak for the hip kinematics (Figure 2). However, Figure 2 clearly shows an offset between the waveforms of the two systems/models and removing this offset resulted in excellent coefficients of multiple correlation for all joints (between 0.956 and 0.977 ). For all joints, the RMSE was between $18^{\circ}$ and $28^{\circ}$ for the waveforms without offset correction and between $5^{\circ}$ and $8^{\circ}$ for the waveforms with offset correction (Table 1).

The RMSE of the calculated ranges of motion in the three joints ranged from $4^{\circ}$ to $9^{\circ}$, while the RMSE of the other discrete parameters ranged from $13^{\circ}$ to $36^{\circ}$ (Table 2). The range of motion of the ankle during stance and swing and of the knee and hip during swing did not differ between the systems/models, while the knee and hip range of motion during stance were significantly smaller when measured with the RehaGait ${ }^{\circledR}$. The offset between the waveforms showed that measurements with the RehaGait ${ }^{\circledR}$ system/model resulted in more ankle plantarflexion, knee extension, and hip extension compared to the reference system/model (Figure 3, Table 4).

\section{Repeatability RehaGait ${ }^{\circledR}$}

The coefficient of multiple correlation of the kinematic waveforms was excellent for all joints for walking (between 0.959 and 0.994). For running, the coefficient of multiple 
244 correlation was very good for the ankle (0.937) and excellent for the knee and hip joint

245 (>0.984). The RMSE of the waveforms measured by the two systems/models was around $3^{\circ}$ 246 for walking and between $3^{\circ}$ and $7^{\circ}$ for running (Table 1).

247 For walking, the RMSE of the discrete parameters between the RehaGait ${ }^{\circledR}$

248 measurements ranged from $0^{\circ}$ to $5^{\circ}$. For running, the RMSE ranged from $1^{\circ}$ to $10^{\circ}$ with the

249 highest RMSE occurring for the ankle range of motion during swing phase (Table 2). Except

250 for the minimal knee angle around foot off during walking, there were no significant

251 differences between the discrete parameters measured during the two measurements with the

252 RehaGait $^{\circledR}$ for both walking and running (Table 3, Table 4). Limits of agreement were larger

253 for running than walking (Figure 3). For the ranges of motion, ICCs were good or excellent

254 for ankle, knee in the second half of the stride, and hip during walking and good or excellent

255 for ankle dorsiflexion, knee in the second half of the stride and hip during running (Figure 3).

\section{Discussion}

The primary aim of this study was to assess the agreement between sagittal plane joint kinematics measured by the inertial sensor system RehaGait ${ }^{\circledR}$ and an optoelectronic system during walking and running. Our results showed that the joint angles measured by the two systems/models were highly correlated, but only after offset correction. The hypothesis that there were no significant differences between discrete kinematic parameters between the two systems/models had to be rejected for most parameters. The secondary aim of the study was to investigate the test-retest repeatability of the kinematic waveforms and the discrete parameters measured by the inertial sensor system/model. The results of this analysis showed very good to excellent correlations between the test and re-test measurements with the

267 RehaGait ${ }^{\circledR}$ system/model and - except for the minimal knee angle around foot off during walking - no significant differences between the discrete parameters measured in the test and re-test sessions. 
Waveforms

The inertial sensor based system/model and optoelectronic system/model used different models to calculate kinematics. Previous research for the knee joint angle showed high correlations and small $\operatorname{RMSE}\left(<3.4^{\circ}\right)$ for walking and running when kinematics were calculated from the segment position data of inertial sensors and marker clusters using the same models (Cooper et al., 2009; Favre et al., 2008; Picerno et al., 2008). The RMSE of the waveforms were smaller than in our study. However, in studies that used independent models to calculate kinematics from inertial systems/models and optoelectronic systems/models very good to excellent correlations but higher RMSEs of $6^{\circ}$ to $11^{\circ}$ with offset correction and of up to $20^{\circ}$ without offset correction were reported (Cloete and Scheffer, 2008; Ferrari et al., 2010b; Takeda et al., 2009). These results are comparable to our results and further emphasize the importance not only of the source of position or movement data (inertial sensor versus cameras) but also of the models used for measuring and calculating joint angles. measured by an inertial sensor system/model and model and an optoelectronic system/model and model used correlation coefficients to compare their similarity (Cloete and Scheffer, 2008; Jaysrichai et al., 2015; Takeda et al., 2009). We used the coefficient of multiple correlation as described by Ferrari (Ferrari et al., 2010a) because it considers the offset between the waveforms, hence, explaining the lower correlation in our study compared to some previous studies. The offset between the waveforms was greater for running than for walking, thus partly explaining the lower coefficients of multiple correlation for running. The

292 RehaGait $^{\circledR}$ model uses boundary conditions (i.e. knee angle is set to $0^{\circ}$ at each initial contact)

293 to deal with the sensor drift during measurements. It is possible, that these boundary

294 conditions are met at a different time point during the stride or at a different joint position for running than for walking, thus increasing the offset between the waveforms. 


\section{Discrete Parameters}

To characterize gait or running patterns, discrete parameters such as minimal and maximal angles or ranges of motion are often calculated. Our results showed that the two systems/models RehaGait ${ }^{\circledR}$ and Vicon ${ }^{\circledR}$ yield significantly different discrete parameters. As described for the waveforms, there was an offset between the systems/models explaining some of the differences in minimal and maximal joint angles. This indicates that the discrete parameters cannot be directly compared between the RehaGait ${ }^{\circledR}$ inertial sensor system $/$ model and optoelectronic Vicon ${ }^{\circledR}$ system/model. Moreover, we also observed systematic differences in the ranges of motion parameters. These could be related to differences in the positioning of sensors and markers and thus in segment positions, and to different definitions of joint axes.

307 For instance, the inertial sensor model uses a technical coordinate system without anatomical information and the PlugIn Gait model uses an anatomical coordinate system. Furthermore, soft tissue movement especially during running might influence marker and sensor positions differently (i.e. due to difference in size or location on the leg), hence increasing differences

311 between the systems/models. Differences in the peak values, but not ranges of motion

312 measured by the two systems/models were greater for running than walking. This is likely

313 related to differences in the offset between the systems.

\section{Repeatability RehaGait ${ }^{\circledR}$}

The coefficients of multiple correlation between the test and re-test RehaGait ${ }^{\circledR}$

317 measurements were very good to excellent which is comparable to the results of a systematic

318 review on the reliability of optoelectronic three-dimensional gait analysis (McGinley et al., 319 2009). For walking the RMSE of the waveforms was around $3^{\circ}$ between the test and re-test

320 measurements, which also lies within the $2^{\circ}$ to $5^{\circ}$ that are reported for optoelectronic gait analyses (McGinley et al., 2009). There were significant differences between the test and re- 
test measurements for many of the discrete parameters. However, for the ranges of motion during walking the limits of agreement were comparable to those reported in the literature for optoelectronic gait analysis (Meldrum et al., 2014). Hence, the repeatability of the RehaGait ${ }^{\circledR}$ system/model for walking is comparable to repeatability of optoelectronic systems/models and suggests a clinically acceptable repeatability. Because the RMSEs were larger for running than walking (especially in the second half of the stride, thus the swing phase), more caution is needed for the interpretation of running measurements, particularly for the swing phase that occurs in the second half of the stride.

\section{Limitations}

For both systems/models, the time of initial contact was determined from the knee

333 flexion/extension angle. Differences in this angle between the systems/models might translate to slight differences in the time point of the initial contact between systems/models and consequently also a time shift in the waveforms. Such a time shift could affect the coefficients of multiple correlation and the joint angles at initial contact, but not range of motion parameters. The RehaGait ${ }^{\circledR}$ and the optoelectronic system/model measured with different sampling rates which could further influence the results on the agreement between the systems/models. Moreover, averaging decreases the influence of possibly not analyzing the same 20 strides of the two systems, because systems were manually started at the same time

341 but not synchronized. The data was collected for walking and running on a treadmill in

342 healthy subjects. It remains to be determined if a comparison of the RehaGait ${ }^{\circledR}$ system/model

343 with an optoelectronic reference system/model during overground walking and running yields

344 similar results. However, treadmill gait analysis is frequently utilized in clinical practice and

345 by therapists and coaches, and hence the results of this study are highly relevant. 
348 This study showed that for healthy subjects the sagittal plane joint kinematic waveforms

349 measured with the RehaGait ${ }^{\circledR}$ inertial sensor system/model are comparable to those of a

350 Vicon ${ }^{\circledR}$ optoelectronic reference system. Because of an offset between the systems $/$ models,

351 discrete parameters cannot be compared directly. The application of this inertial sensor system

352 is easy and less time consuming than that of the optoelectronic system. The repeatability of

353 the RehaGait ${ }^{\circledR}$ system/model was better for walking than running. Our results showed that the

354 RehaGait $^{\circledR}$ system/model provides important and relevant information on gait patterns with

355 clinically acceptable repeatability for treadmill walking and the stance phase, but not the

356 swing phase of running.

357

\section{Conflict of interest statement}

The authors declare no conflict of interest.

\section{Acknowledgement}

The authors thank Nils Altrogge for his assistance in data collection. This study was

funded in part by the Swiss National Science Foundation (SNSF \#32003B_159871/1).

\section{References}

Cloete, T., Scheffer, C., 2008. Benchmarking of a full-body inertial motion capture system for clinical gait analysis. In: 2008 30th Annual International Conference of the IEEE Engineering in Medicine and Biology Society: 2008: 4579-4582.

Cooper, G., Sheret, I., McMillian, L., Siliverdis, K., Sha, N., Hodgins, D., Kenney, L., Howard, D., 2009. Inertial sensor-based knee flexion/extension angle estimation. 42, $2678-2685$. 
Donath, L., Faude, O., Lichtenstein, E., Nüesch, C., Mündermann, A., 2016. Validity and reliability of a portable gait analysis system for measuring spatiotemporal gait characteristics: comparison to an instrumented treadmill. 13, 1 .

Favre, J., Jolles, B.M., Aissaoui, R., Aminian, K., 2008. Ambulatory measurement of 3D knee joint angle. 41, 1029-1035.

Fellin, R.E., Rose, W.C., Royer, T.D., Davis, I.S., 2010. Comparison of methods for kinematic identification of footstrike and toe-off during overground and treadmill running. 13, 646-650.

Ferrari, A., Cutti, A.G., Cappello, A., 2010a. A new formulation of the coefficient of multiple correlation to assess the similarity of waveforms measured synchronously by different motion analysis protocols. $31,540-542$.

Ferrari, A., Cutti, A.G., Garofalo, P., Raggi, M., Heijboer, M., Cappello, A., Davalli, A., 2010b. First in vivo assessment of "Outwalk": a novel protocol for clinical gait analysis based on inertial and magnetic sensors. 48, 1-15.

Fleiss, J.L., 1986. Design and analysis of clinical experiments. John Wiley \& Sons, NewYork. Hamacher, D., Hamacher, D., Taylor, W.R., Singh, N.B., Schega, L., 2014. Towards clinical application: repetitive sensor position re-calibration for improved reliability of gait parameters. Gait Posture 39, 1146-1148.

Jaysrichai, T., Suputtitada, A., Khovidhungij, W., 2015. Mobile sensor application for kinematic detection of the knees. 39, 599-608.

Kadaba, M.P., Ramakrishnan, H.K., Wootten, M.E., 1990. Measurement of lower extremity kinematics during level walking. Journal of Orthopaedic Research 8, 383-392.

McGinley, J.L., Baker, R., Wolfe, R., Morris, M.E., 2009. The reliability of three-dimensional kinematic gait measurements: a systematic review. 29, 360-369. 
Meldrum, D., Shouldice, C., Conroy, R., Jones, K., Forward, M., 2014. Test-retest reliability of three dimensional gait analysis: Including a novel approach to visualising agreement of gait cycle waveforms with Bland and Altman plots. 39, 265-271.

Picerno, P., Cereatti, A., Cappozzo, A., 2008. Joint kinematics estimate using wearable inertial and magnetic sensing modules. 28, 588-595.

401 Schwesig, R., Kauert, R., Wust, S., Becker, S., Leuchte, S., 2010. Reliabilitätsstudie zum Ganganalysesystem RehaWatch/Reliability of the novel gait analysis system RehaWatch. 55, 109-115.

Sprager, S., Juric, M.B., 2015. Inertial sensor-based gait recognition: a review. 15, 22089-

405 22127.

406

Takeda, R., Tadano, S., Natorigawa, A., Todoh, M., Yoshinari, S., 2009. Gait posture

407 estimation using wearable acceleration and gyro sensors. 42, 2486-2494.

408

409 
Table 1: Root mean square error (RMSE) (1 standard deviation) between the kinematic waveform data measured by the RehaGait ${ }^{\circledR}$ and the reference system without and with offset correction, respectively and within the two sessions measured with the RehaGait ${ }^{\circledR}$ system for treadmill walking and running

\begin{tabular}{lccc}
\hline & $\begin{array}{c}\text { Between RehaGait } \\
\text { and Vicon without } \\
\text { offset correction }\end{array}$ & $\begin{array}{c}\text { Between RehaGait }^{(B)} \\
\text { and Vicon with offset } \\
\text { correction }\end{array}$ & Within RehaGait $^{(B)}$ \\
\hline Walking & & & \\
RMSE ankle & $4.5(2.1)$ & $2.5(0.9)$ & $2.7(1.7)$ \\
RMSE knee & $7.6(2.6)$ & $5.0(1.7)$ & $3.1(1.8)$ \\
RMSE hip & $9.6(3.0)$ & $3.3(0.8)$ & $3.0(2.5)$ \\
Running & & & \\
RMSE ankle & $17.7(5.4)$ & $5.4(3.6)$ & $6.7(4.1)$ \\
RMSE knee & $17.9(4.4)$ & $7.8(3.5)$ & $5.3(3.1)$ \\
RMSE hip & $27.6(3.2)$ & $5.3(2.2)$ & $3.8(2.4)$ \\
\hline
\end{tabular}


Table 2: Root mean square error of the discrete parameters between the RehaGait ${ }^{\circledR}$ and Vicon ${ }^{\circledR}$ system and between the test and re-test measurement with the RehaGait ${ }^{\circledR}$ system.

\begin{tabular}{|c|c|c|c|c|}
\hline & \multicolumn{2}{|c|}{ Walking } & \multicolumn{2}{|c|}{ Running } \\
\hline & $\begin{array}{c}\text { Between } \\
\text { RehaGait }^{\circledR} \\
\text { and Vicon }\end{array}$ & $\begin{array}{c}\text { Within } \\
\text { RehaGait }^{\circledR}\end{array}$ & $\begin{array}{c}\text { Between } \\
\text { RehaGait }^{\circledR} \\
\text { and Vicon }\end{array}$ & $\begin{array}{c}\text { Within } \\
\text { RehaGait }^{\circledR}\end{array}$ \\
\hline Ankle angle at initial contact & 4.2 & 2.5 & 14.4 & 6.1 \\
\hline first minimal ankle angle & 5.4 & 0.6 & 17.5 & 2.1 \\
\hline Maximal ankle angle & 4.6 & 2.0 & 19.1 & 3.7 \\
\hline second minimal ankle angle & 5.2 & 3.2 & 18.5 & 10.1 \\
\hline Ankle dorsiflexion range of motion & 4.4 & 1.8 & 5.3 & 2.8 \\
\hline Ankle plantarflexion range of motion & 4.0 & 2.6 & 7.1 & 10.4 \\
\hline Knee angle at initial contact & 9.9 & 0.5 & 19.3 & 1.4 \\
\hline first maximal knee angle & 10.1 & 3.3 & 20.0 & 5.4 \\
\hline Minimal knee angle & 5.3 & 3.6 & 13.2 & 4.9 \\
\hline second maximal knee angle & 7.1 & 4.3 & 19.8 & 8.8 \\
\hline $\begin{array}{l}\text { Knee range of motion (first half } \\
\text { stride) }\end{array}$ & 3.7 & 3.1 & 5.7 & 3.9 \\
\hline $\begin{array}{l}\text { Knee range of motion (second half } \\
\text { stride) }\end{array}$ & 8.4 & 4.1 & 7.6 & 9.1 \\
\hline Hip angle at initial contact & 14.6 & 4.1 & 36.1 & 3.5 \\
\hline first maximal hip angle & 12.8 & 3.5 & 33.2 & 2.7 \\
\hline Minimal hip angle & 6.0 & 3.9 & 25.7 & 5.3 \\
\hline second maximal hip angle & 9.8 & 3.7 & 25.1 & 3.8 \\
\hline Hip range of motion (first half stride) & 7.6 & 2.3 & 8.6 & 4.0 \\
\hline $\begin{array}{l}\text { Hip range of motion (second half } \\
\text { stride) }\end{array}$ & 4.6 & 1.9 & 4.2 & 3.9 \\
\hline
\end{tabular}




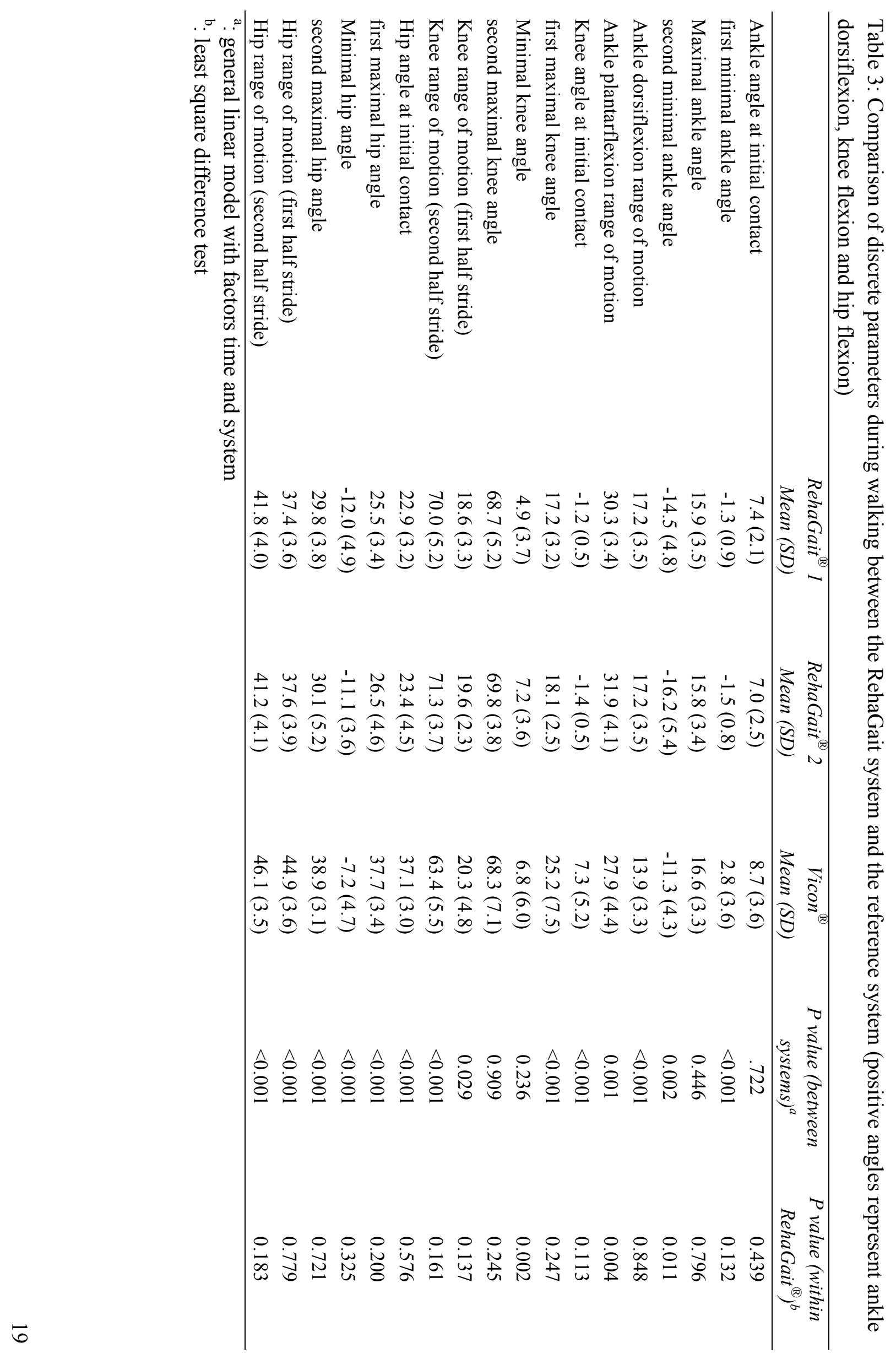




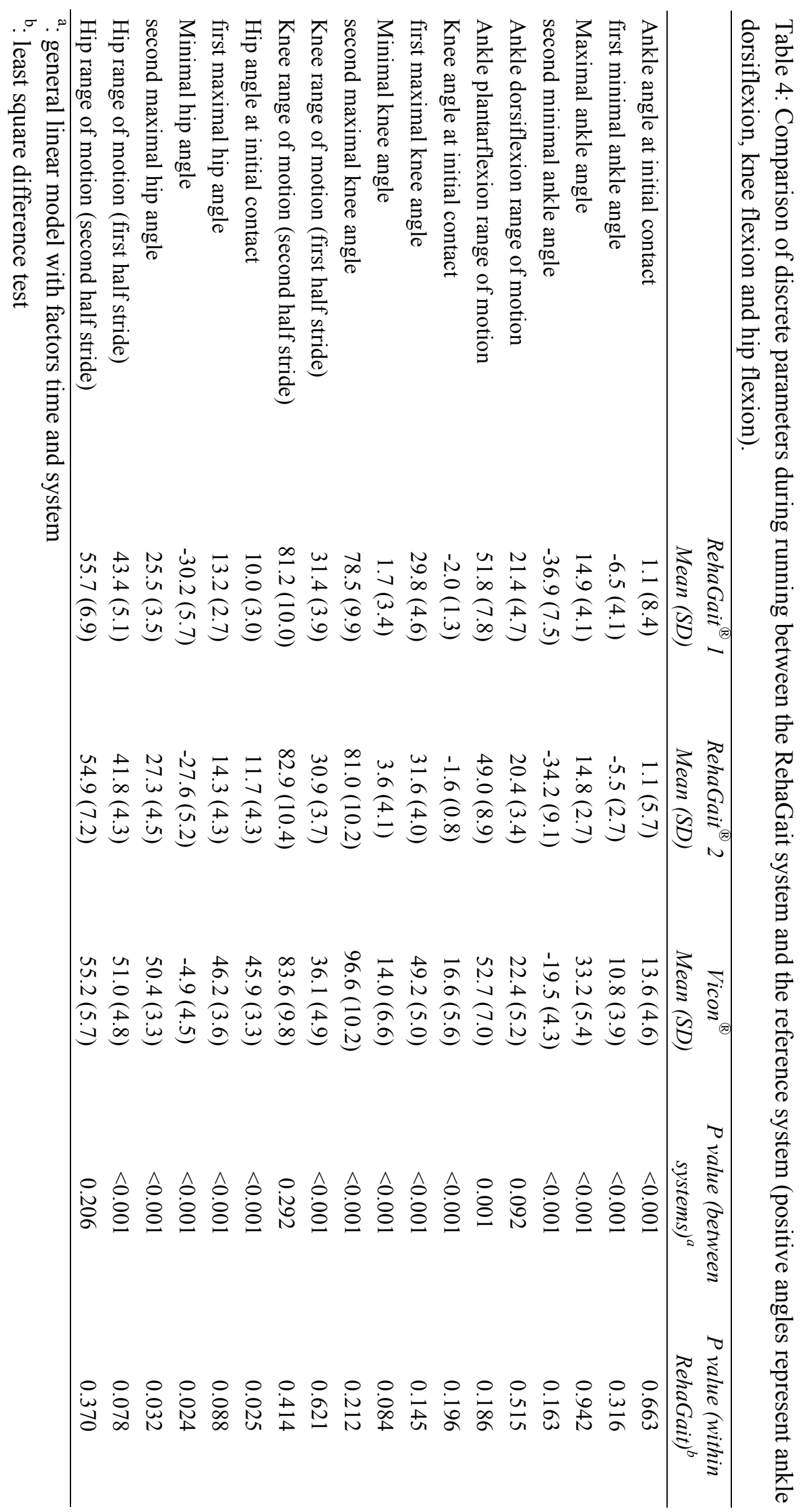




\section{Figure Captions}

Figure 1: A) Inertial sensor with elastic strap; B) Placement of the inertial sensors laterally on the foot (below lateral malleolus) and the shank (lower third); C) Dorsal view of the placement of the inertial sensors on the foot, shank, thigh (middle) and sacrum.

Figure 2: Comparison between mean joint angles of the 20 subjects during walking (left column) and running (right column) measured by the RehaGait ${ }^{\circledR}$ (dashed line) and the reference system (solid line). The grey area indicates the mean $\pm 95 \%$ confidence interval difference between the two systems. For each joint and conditions the coefficient of multiple correlation $(\mathrm{CMC})$ is indicated in the respective graph.

Figure 3: Bland-Altman plots for the ranges of motion (ROM) of the ankle, knee and hip joint during the stance phase for the test re-test comparison of walking (left column) and running (right column). Each graph presents the mean difference (solid line) and 1.96-fold standard deviation of the difference (dashed lines) between the two measurements. Intraclass correlation coefficients (ICC) between the measurements are indicated in the titles of each angle. 


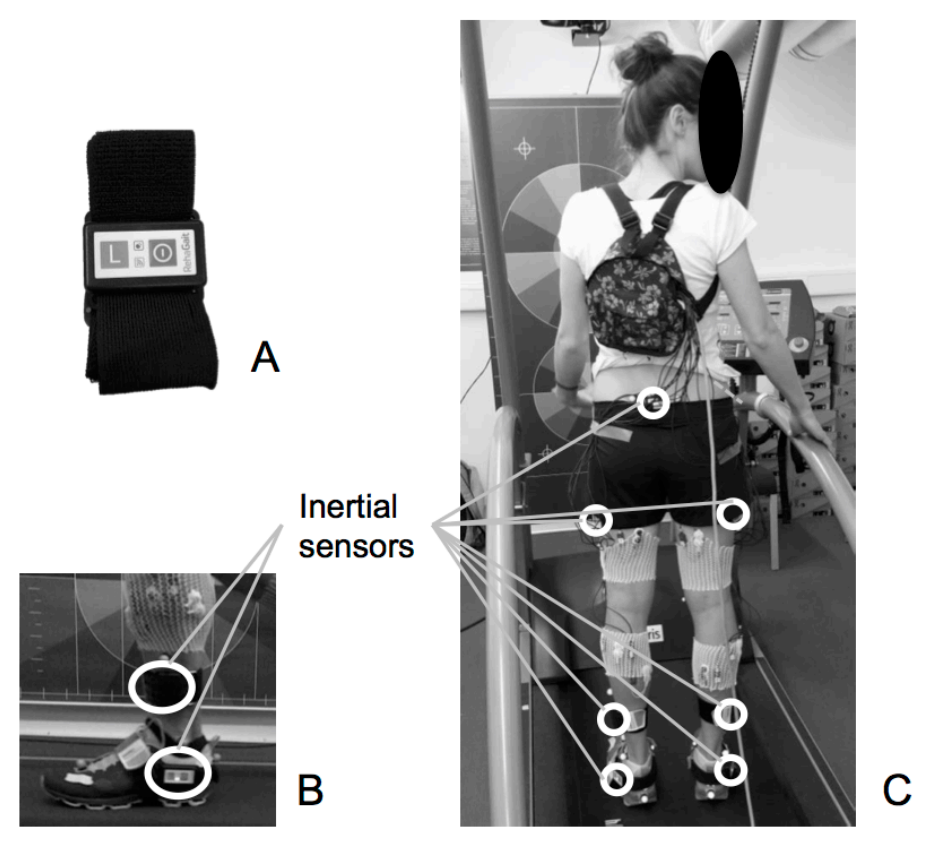

Figure 1 

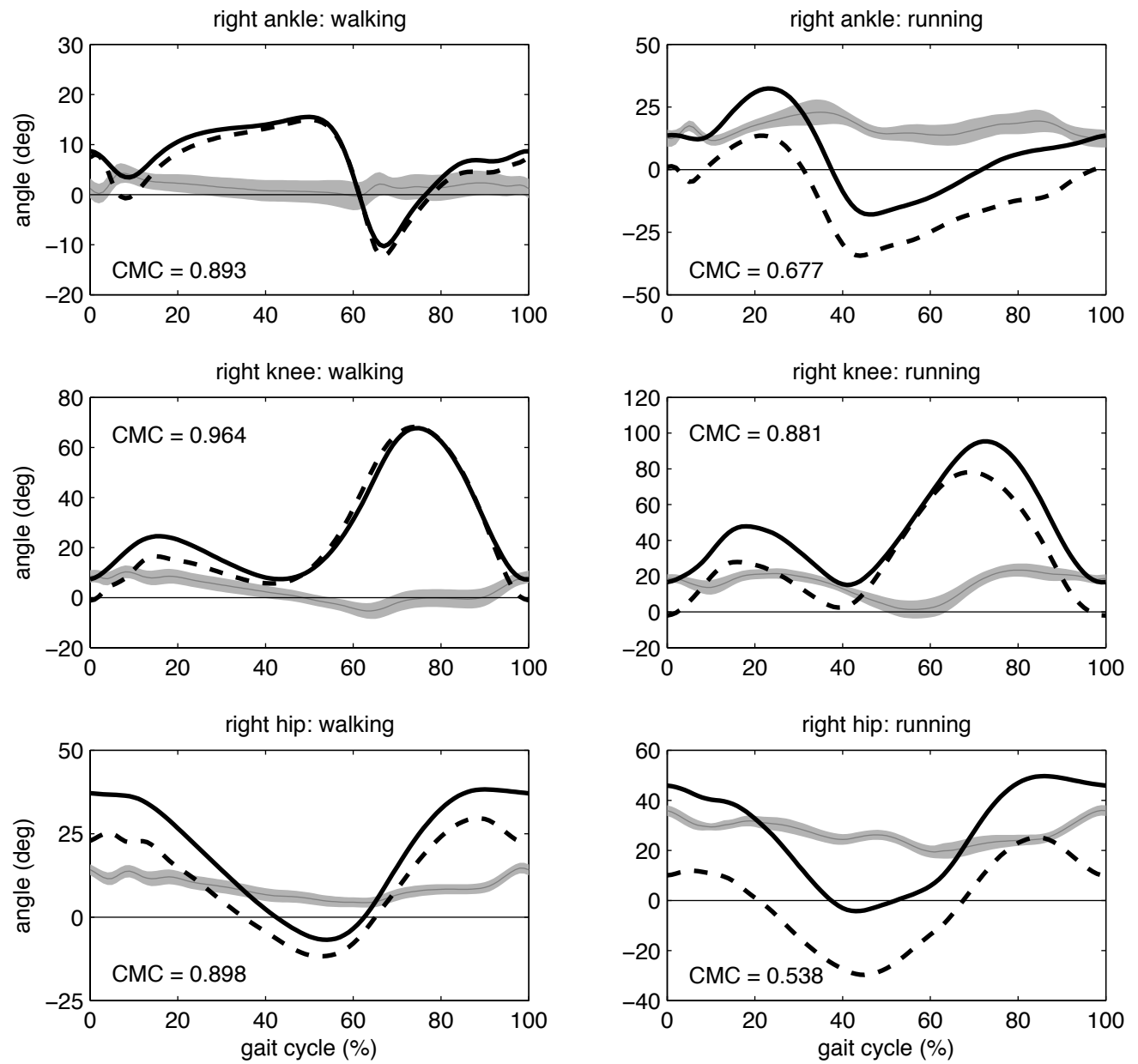

Figure 2 

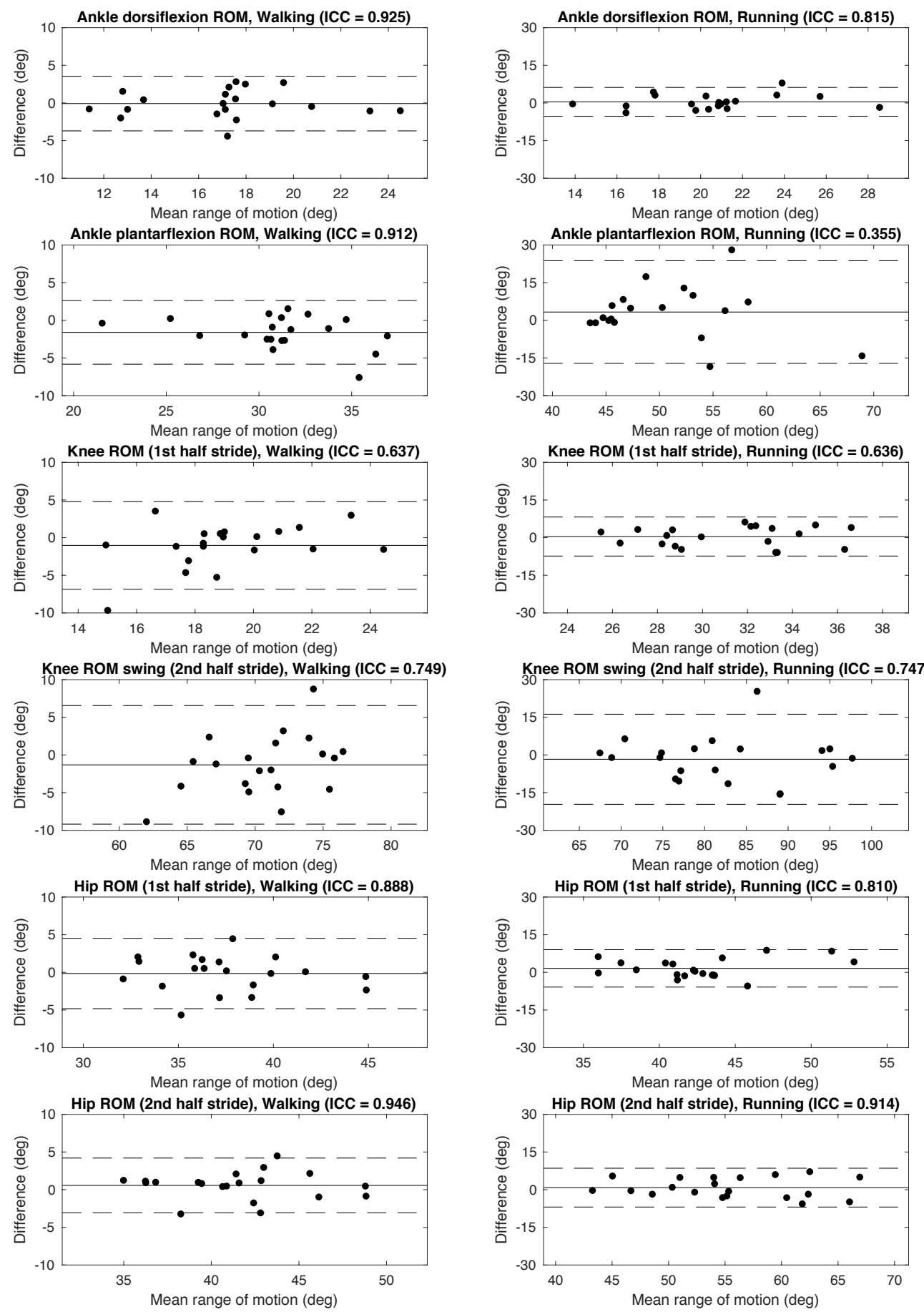

Figure 3 